\title{
Optical trapping with Bessel beams generated from semiconductor lasers
}

\author{
G S Sokolovskii, ${ }^{1}$ V V Dudelev, ${ }^{1}$ S N Losev, ${ }^{1}$ K K Soboleva, ${ }^{1,2}$ \\ A G Deryagin, ${ }^{1}$ V I Kuchinskii, ${ }^{1,3}$ W Sibbett, ${ }^{4}$ E U Rafailov ${ }^{5}$ \\ ${ }^{1}$ Ioffe Physical-Technical Institute, St.Petersburg, Russia \\ ${ }^{2}$ Saint-Petersburg State Polytechnical University, St.Petersburg, Russia \\ ${ }^{3}$ Saint-Petersburg State Electrotechnical University “LETI”, St.Petersburg, Russia \\ ${ }^{4}$ School of Physics and Astronomy, University of St.Andrews, St.Andrews, UK \\ ${ }^{5}$ Aston Institute of Photonic Technologies, Aston University, Birmingham, UK \\ gs@mail.ioffe.ru
}

\begin{abstract}
In this paper, we study generation of Bessel beams from semiconductor lasers with high beam propagation parameter $\mathrm{M}^{2}$ and their utilization for optical trapping and manipulation of microscopic particles including living cells. The demonstrated optical tweezing with diodegenerated Bessel beams paves the way to replace their vibronic-generated counterparts for a range of applications towards novel lab-on-a-chip configurations.
\end{abstract}

\section{Introduction}

Optical trapping and tweezing technique is based on the forces that arise as a consequence of the law of conservation of momentum in the absorption, reflection and refraction of the laser beam at the particle [1]. A prerequisite for successful optical manipulation, i.e. reliable trapping of microparticles, is the high gradient of the optical power density. In other words, in order to trap the microparticle, one must sharply focus the laser light. This significantly reduces the workspace due to diffraction: tighter focusing results in faster divergence of radiation, and the problem can not be resolved in terms of the Gaussian optics. However, as was shown in the seminal work by Durnin [2] (and earlier by Zeldovich et al. [3]) diffractive divergence can be almost completely eliminated with a special class of non-diffracting light fields called Bessel beams. This class of propagation-invariant beams is generated through the interference of the conically converging rays taking place when a collimated Gaussian beam transits a cone-shaped lens (axicon [4]). The result of such interference is a bright spot surrounded by concentric fringes. The radial intensity profile of this distribution can be described by a zero-order Bessel function of the first kind as illustrated in Fig.1. The central lobe diameter of the Bessel beam is determined by the axicon apex angle and can be of the order of the optical wavelength. Practically, Bessel beams exhibit a finite propagation distance, which depends on the cross-sectional diameter of the initial collimated beam. Since Bessel beams maintain their focus along the line, the position of manipulated object can be significantly varied, thus making micromanipulation systems flexible and more attractive for practical implementations. The utilization of Bessel beams also opens new horizons in microporation [5], manipulation of micromachines [6] micro-fabrication [7,8] and various 'lab-on-a-chip' applications [9,10].

Until recently, it was believed that generation of Bessel beams is only possible with highly coherent light sources, such as gas and solid-state lasers, but last years a number of publications reported on formation of Bessel beams from semiconductor light sources [11-13], including various types of edgeemitting laser diodes [11,12], vertical-cavity surface-emitting lasers (VCSELs) [11] and vertical externalcavity surface-emitting lasers (VECSELs) [13], and even light-emitting diodes (LEDs) [11]. 


\section{Generation of Bessel beams from semiconductor lasers with high beam propagation parameter} Typically, the 'quality' of the laser beam can be described by the beam propagation parameter $\mathrm{M}^{2}[14$, 15], which is defined as the ratio of the beam divergence to that of the 'ideal' Gaussian beam (i.e. the beam with $\mathrm{M}^{2}=1$ ) corresponding to the diffraction limit. Parameter $\mathrm{M}^{2}$ allows the description of the quasi-Gaussian beams with mathematics developed for the 'ideal' Gaussian beams with simple replacement $\lambda \rightarrow \mathrm{M}^{2} \lambda$, i.e. with $\mathrm{M}^{2}$-fold numerical increase of the wavelength.

$$
N A=\frac{M^{2} \lambda}{\pi \omega_{0}}
$$

where $N A$ is the numerical aperture, $\lambda$ is the wavelength, and $\omega_{0}$ is the beam waist size. With high parameter $\mathrm{M}^{2}$ of the initial quasi-Gaussian beam typical for the laser diodes, the diameter of the central lobe of the Bessel beam gradually increases with propagation distance due to the high divergence of the forming beam. This leads to limitation of the propagation distance $z_{\mathrm{B}}$ of the generated Bessel beam (see Fig. 1). Here, one should bear in mind that without divergence of the forming beam taken into account, the propagation distance $Z_{\mathrm{B} 0}$ of the Bessel beam is defined by the geometrical parameters of the optical scheme [16].

In this paper, we assume that the initial quasi-Gaussian beam is coaxial with the axicon and collimated in the plane of its apex. Therefore in the paraxial approximation, the diameter of the central lobe of the Bessel beam, which is normally given by $d(z)=2.4 \lambda /(\pi(n-1) \cos (\alpha / 2))$, in view of (1) transforms to [17]:

$$
d(z) \approx \frac{2.4 \lambda}{\pi(n-1) \cos \alpha / 2}\left[1+\left(\frac{M^{2} \lambda z}{\pi \omega_{0}^{2}}\right)^{2}\right]
$$

where $\alpha$ is the apex angle of the axicon, $n$ is the refractive index, and $z$ is the axial distance. Equation (2) greatly simplifies the calculation of the propagation distance of the Bessel beam limited by the divergence of the forming beam [18]. Let's define the propagation distance $z_{\mathrm{B}}$ of the Bessel beam as the distance at which the transverse size of the central lobe increases $\sqrt{2}$-fold (similar to the definition of the Rayleigh range for the Gaussian beam).

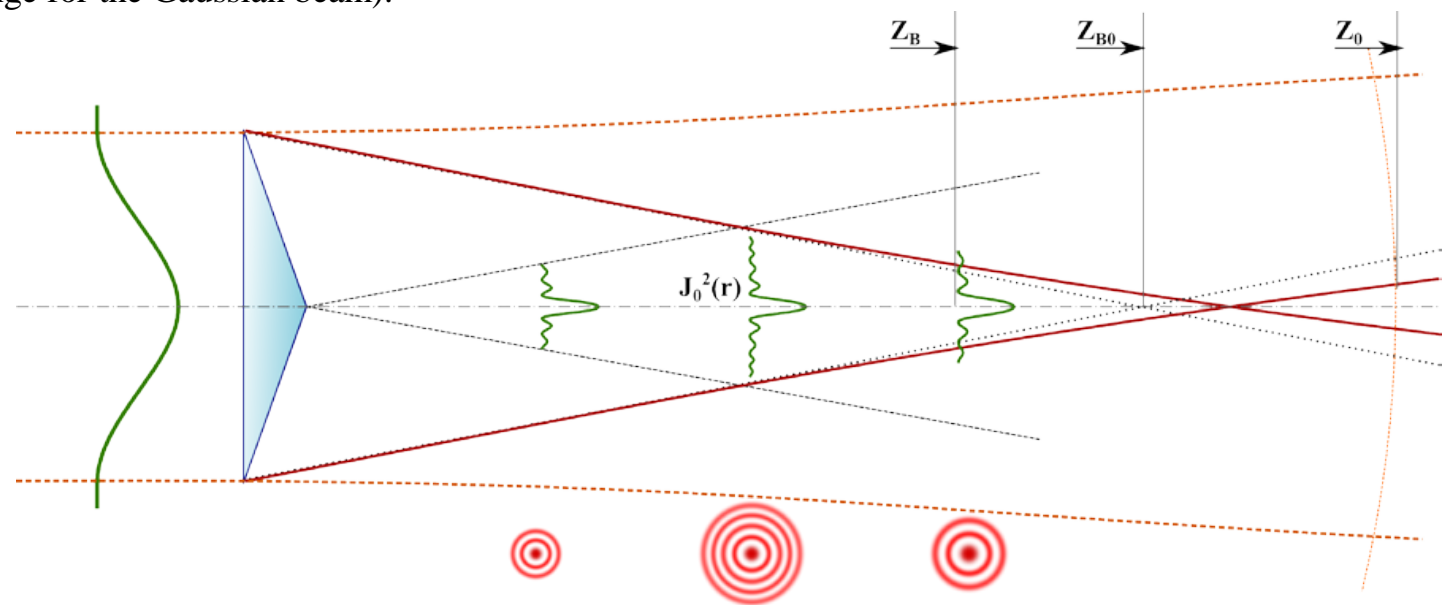

Figure. 1. Propagation of the Bessel beam formed from a quasi-Gaussian beam with a high parameter $\mathrm{M}^{2}: \mathrm{z}_{\mathrm{B}}$ is the Bessel beam propagation distance due to the beam divergence; $\mathrm{z}_{\mathrm{B} 0}$ is the geometrical propagation distance; $z_{0}$ is the Rayleigh range.

After simple transformations [16,17] equation (2) yields the propagation length of the Bessel beam generated from the quasi-Gaussian beam with high $\mathrm{M}^{2}$ :

$$
z_{B} \approx \frac{2 \omega_{0}^{2}}{M^{2} \lambda}
$$

From (3) one can easily see that, despite the negative influence of the high beam propagation parameter, almost any required propagation length of the Bessel beam is achievable by increasing the radius of the initial quasi-Gaussian beam. Moreover, even with tight collimation of the quasi-Gaussian beam required for the power-demanding applications, the satisfying propagation length of the Bessel beam can be achieved as we confirm in the experiments below. 


\section{Optical trapping with Bessel beams generated from semiconductor lasers}

In this paper, we demonstrate the use of the Bessel beams generated from the laser diodes for optical trapping and manipulation of microscopic particles including living cells which build on our recent results reported elsewhere [19]. A scheme of the experimental setup is shown in Fig. 2.

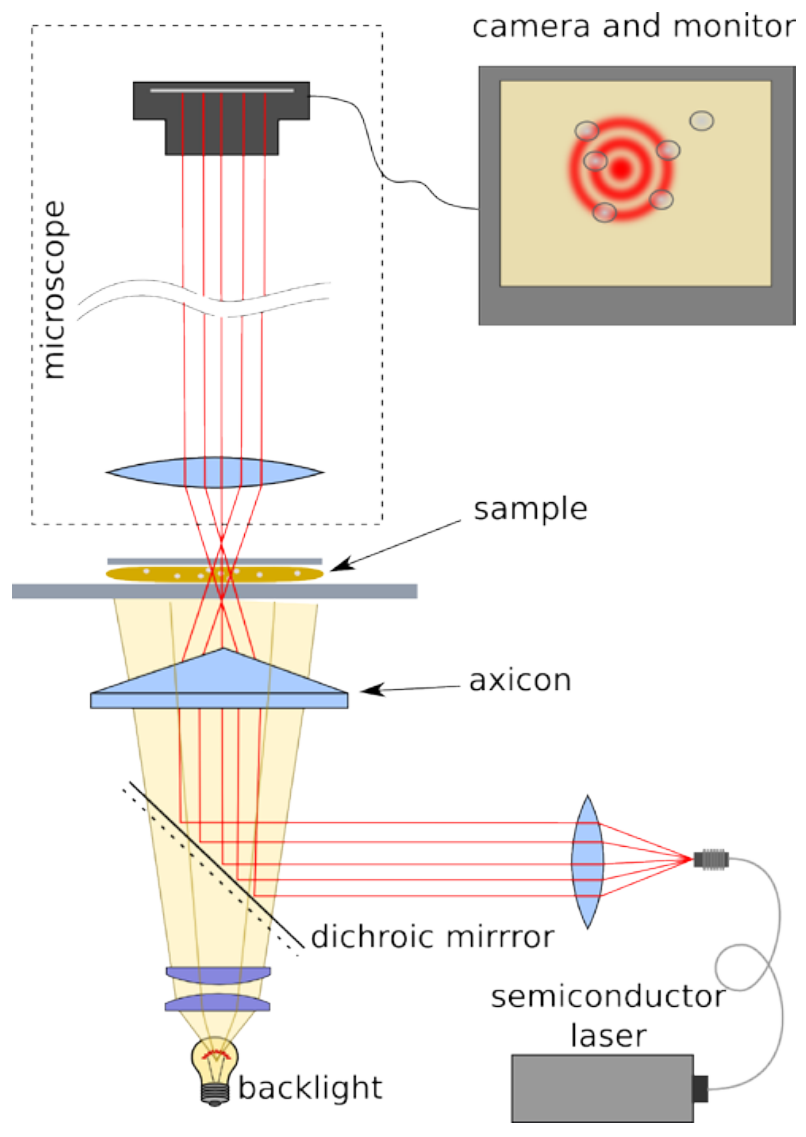

Figure 2. Scheme of the experimental setup for optical trapping and manipulation of microscopic particles with Bessel beams generated the laser diode.

For generation of Bessel beams in our experiments we used the fiber-coupled edge-emitting semiconductor laser with the output power up to $600 \mathrm{~mW}$ and the emission spectrum centered at $1065 \mathrm{~nm}$. The output laser beam was collimated and refocused by the optical system including a set of interchangeable microscope lenses with magnification ranging from x8 to x60 and a dichroic mirror transmitting backlight (see Fig.2). The Bessel beam was generated with the axicon of $160^{\circ}$ apex angle, which determined the transverse size of the central lobe of $\sim 7 \mu \mathrm{m}$. The size of the Bessel beam features was measured with a graded transparent grid (so-called 'graticule') with scale divisions of $10 \mu \mathrm{m}$ and the total length of $1 \mathrm{~mm}$ graded scale. The graticule was placed in the focal plane of the microscope lens in order to enable detection of the Bessel beam passing through the scaled region. This ensured precise control of magnification of the microscope detection system. The optical power in the central lobe of the Bessel beam with losses in the optical scheme taken into account was up to $20 \mathrm{~mW}$.

The mechanical control for the optical trapping experiments was enabled by the high-precision micropositioners introduced into the optical scheme and a commercial microscope with a video-recording CCD camera. The micro-objects for the study were polystyrene beads (10 $\mu \mathrm{m}$ mean diameter) in a water solution as the high refractive index objects and water bubbles in oil as the low-index particles. The solution containing the micro-objects was placed on a glass slide covered with a cover glass and controlled with a micropositioner. Dichroic mirror enabled backlight illumination (see Fig. 2) .

We also studied the optical trapping of living red cells of rat blood. In the assessment, blood was dissolved in water with heparin added to prevent clotting. The mean size of the cells was approximately $5 \mu \mathrm{m}$ (measured with graticule, similar to the measurement of the size of the features of the Bessel beam). The video in Fig. 3 demonstrates the two-dimensional optical trapping and manipulation of a living red cell of rat blood in the central lobe of the Bessel beam generated from the semiconductor laser. No damage of the living cells was detected in the available optical power range. 


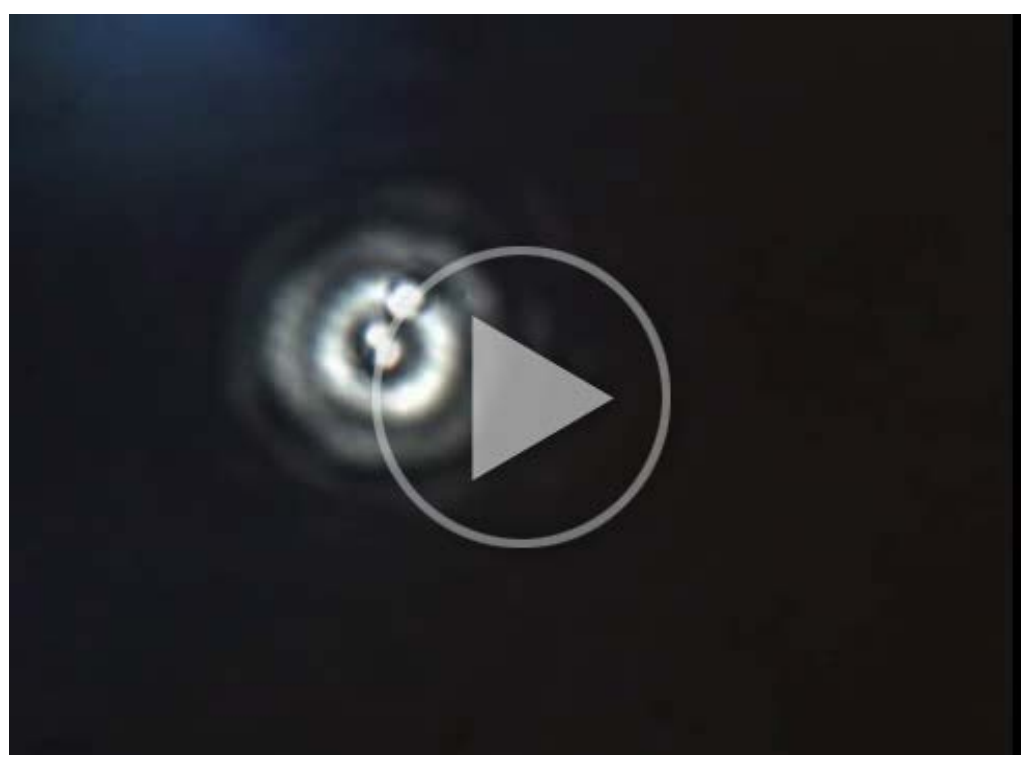

Figure 3. Optical trapping of a living red blood cell in the central lobe of the Bessel beam generated from the semiconductor laser.

Our assessments to date demonstrated very good potential for the Bessel beams generated from semiconductor lasers to replace their gas and solid-state laser-generated counterparts in applications relating to optical trapping and manipulation of microparticles including living biological micro-objects. The most attractive of these are so-called 'lab-on-a-chip' configurations. The important advantage in this sense is the recently demonstrated possibility of superfocusing of the laser diode beams with high parameter $\mathrm{M}^{2}$ by generation of Bessel beams $[18,20]$. Combination of compactness and efficiency of semiconductor lasers with superfocusing and suppressed divergence of the beam opens up tremendous prospects for their 'lab-on-a-chip' applications.
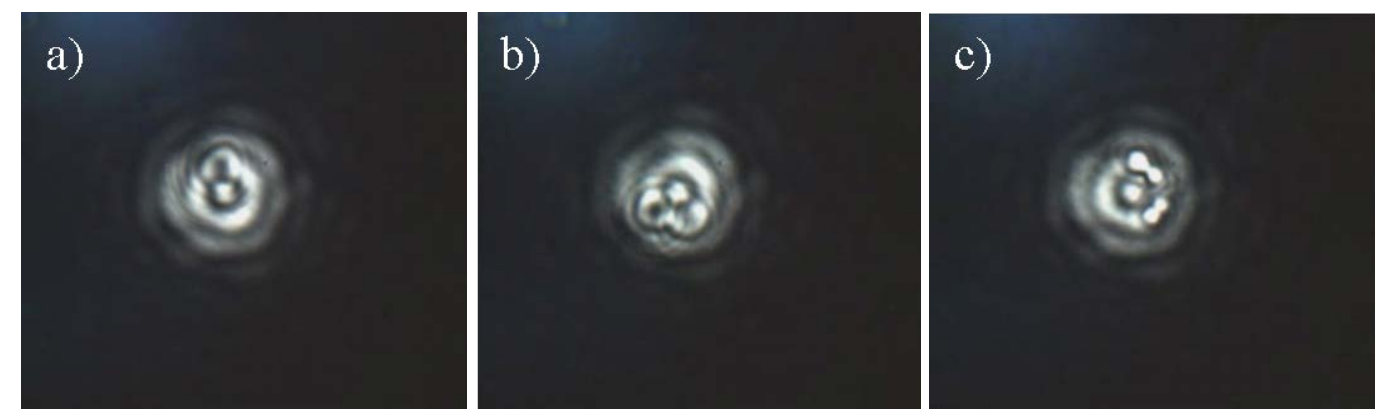

Figure 4. Trapping of multiple rat blood erythrocytes simultaneously using a Bessel beam: 2 captured particles (a), 3 captured particles (b), and 5 captured particles (c).

In our experiments, was also demonstrated the ability to capture and manipulate several microscopic objects with a single Bessel beam of sufficient optical power simultaneously. In Fig. 4 one can see one, two and four beads trapped in the first fringe of the Bessel beam in addition to the one trapped in its central lobe.

\section{Conclusion}

The properties of Bessel beams generated from laser diodes with high beam propagation parameter $\mathrm{M}^{2}$ and their applications to optical tweezers are discussed. We have shown that the propagation length of such Bessel beams is defined by the divergence of the initial quasi-Gaussian beam rather than the 
parameters of the optical system. We have also demonstrated the two-dimensional optical trapping and manipulation of microparticles and small biological objects with diode-generated Bessel beams.

\section{References}

[1] Ashkin A 1970 Phys. Rev. Lett. vol 24 p 156.

[2] Durnin J 1987 J. Opt. Soc. Am. vol A4 p 651.

[3] Zel’dovich B Ya, Pilipetskii N F 1966 Izvestia VUZov Radiophysics vol 9 p 95.

[4] McLeod J H 1954 J. Opt. Soc. Am. vol 44 p 592.

[5] Tsampoula X, Garces-Chavez V, Comrie M, Stevenson D J, Agate B, Brown C T A, GunnMoore F, Dholakia K 2007 Appl. Phys. Lett. vol 91053902.

[6] Larsen A E, Grier D G 1997 Nature vol 385, p 230.

[7] Friese M J, Rubinsztein-Dunlop H, Gold J, Hagberg P, Hanstorp D 2001 Appl. Phys. Lett. vol 78 p 547

[8] Amako J, Sawaki D, Fujii E 2003 J. Opt. Soc. Am. vol 20 p 2562

[9] Garcés-Chávez V, McGloin D, Melville H, Sibbett W, Dholakia K 2002 Nature vol 419, p 145.

[10] Rendall H A, Marchington R F, Praveen B B, Bergmann G, Arita Y, Heisterkamp A, Gunn-Moorec F J, Dholakia K 2012 Lab on a Chip vol 12, p 4816.

[11] Sokolovskii G S, Dudelev V V, Losev S N, Zolotovskaya S A, Deryagin A G, Kuchinskii V I, Rafailov E U, Sibbett W 2008 Tech. Phys. Lett. vol 34(12) p 1075.

[12] Sokolovskii G S, Dudelev V V, Losev S N, Deryagin A G, Vinokurov D A, Lyutetskiy A V, Pikhtin N A, Slipchenko S O, Tarasov I S, Zolotovskaya S A, Rafailov E U, Kuchinskii V I, Sibbett W 2010 Tech. Phys. Lett., vol 36(1) p 9.

[13] Sokolovskii G S, Butkus M, Losev S N, Dudelev V V, Deryagin A G, Kuchinskii V I, Sibbett W, Rafailov E U 2012 Proc. SPIE Int. Soc. Opt. Eng., vol 8242, 82420T.

[14] Siegman A E 1998 OSA Annual Meeting.

[15] ISO 11146

[16] Sokolovskii G S, Zolotovskaya S A, Losev S N, Dudelev V V, Deryagin A G, Kuchinskii V I, Sibbett W, Rafailov E U 2011 Proc. SPIE Int. Soc. Opt. Eng., 7919, 79190J

[17] Sokolovskii G S, Dudelev V V, Losev S N, Butkus M, Soboleva K K, Sobolev A I, Deryagin A G, Kuchinskii V I, Sibbett W, Rafailov E U 2013 Quant. El. vol 43 (5) p 423

[18] Sokolovskii G S, Dudelev V V, Losev S N, Deryagin A G, Kuchinskii V I, Sibbett W, Rafailov E U 2012 Tech. Phys. Lett. vol 38(5), p 402.

[19] Sokolovskii G S, Losev S N, Soboleva K K, Dudelev V V, Deryagin A G, Sibbett W, Kuchinskii V I, Rafailov E U 2014 Tech. Phys. Lett. vol 40(6), p 495.

[20] Sokolovskii G S, Melissinaki V, Dudelev V V, Losev S N, Soboleva K K, Kolykhalova E D, Deryagin A G, Kuchinskii V I, Viktorov E A, Farsari M, Sibbett W, Rafailov E U 2014 Proc. SPIE Int. Soc. Opt. Eng. vol 9134, 913457. 